\title{
Spectrum of viral skin infections in patients attending the dermatology clinic at King Fahd Hospital of the University in Alkhobar, Kingdom of Saudi Arabia during the period 2010-2014
}

\author{
Al Anood A. Al Thukair, Deema H. Sallout, Dina W. Abdulghani, Dina T. Al Afandi, \\ Hawra A. Al Johi, Hessa O. Al Otaibi, Lina M. Abu Gharara, Iqbal A. Bukhari
}

Dermatology Department, College of Medicine, Imam Abdulrahman AlFaisal University (University of Dammam) and King Fahd Hospital of the University, Alkhobar, Kingdom of Saudi Arabia

Corresponding author: Dr. Hawra A. Al Johi, E-mail: aljohi.hawra@hotmail.com

\begin{abstract}
Introduction: Viral skin infections are an important disease group in the dermatology field. Many factors play a role in the incidence of skin diseases. There are only a few reports of surveys conducted on the prevalence of viral infections in Saudi Arabia. Our study is the first in the gulf region which can serve as a good reference for planning therapeutic healthcare measures. Objectives: To determine the prevalence of viral skin infections in patients who attended the dermatology clinic at King Fahad Hospital of the University (KFHU) in Alkhobar, Kingdom of Saudi Arabia during a period of five years. Materials and Methods: A retrospective study conducted at the outpatient clinic of the Dermatology Department of KFHU for the period between January 2010 and December 2014. Patients age, gender, diagnosis were documented in a data sheet and analyzed using SPSS V.16. Results: The total number of patients seen during that period was $4798,25.8 \%$ were in the age group of $21-30$ years, $19.5 \%$ were in the age group of $11-20$ years, and $14.1 \%$ were in the age group of 2-10 years. The most commonly encountered skin diseases were acne (16.5\%) followed by eczema (15\%), pigmentary disorders (9.6\%) and viral infections (7.9\%). A variety of viral skin infections were diagnosed including warts, herpes labialis, herpes genitalis, chicken pox and molluscum contagiosum. Warts comprised $72.5 \%$ of the total reported viral infections while Chicken pox and herpes genitalis were the least common reported diseases. Discussion: The most commonly encountered disease group among the 4798 patients seen at KFHU was acne (16.5\%). The second was dermatitis and eczema (15\%). Pigmentary disorders came in third place (9.6\%), and viral infections followed in fourth place (7.9\%). Compared to previous studies in Saudi Arabia, ours showed the lowest prevalence. Warts comprised $72 \%$. Molluscum contagiosum (11.4\%) and Herpes zoster 7.7\%. The least commonly encountered viral diseases were chicken pox and herpes genitalis. Conclusion: Viral skin diseases are one of the major health problems in the dermatology specialty. Further studies should be performed to control these conditions.
\end{abstract}

Key words: Viral infection; herpes; wart; molluscum contagiosum

\section{INTRODUCTION}

Viral skin infections are an important disease group in the dermatology field. Many factors play a role in the incidence of skin diseases. These include geographic area, climate, socioeconomic status, genetics, gender, age, personal habits and internal factors, such as age, gender, and heredity which explains the variable skin diseases reported in different regions [1]. This study was conducted the dermatology clinic of King Fahd Hospital of the University (KFHU) in Alkhobar city in the eastern region of Kingdom of Saudi Arabia. The eastern region

\footnotetext{
How to cite this article: Al Thukair AA, Sallout DH, Abdulghani DW, Al Afandi DT, Al Johi HA, Al Otaibi HO, Abu Gharara LM, Bukhari IA. Spectrum of viral skin infections in patients attending the dermatology clinic at King Fahd Hospital of the University in Alkhobar, Kingdom of Saudi Arabia during the period 2010 - 2014. Our Dermatol Online. 2017;8(3):260-263

Submission: 20.10.2016; Acceptance: 02.01.2017

DOI: 10.7241 /ourd.20173.75
} 
resembles the climate of a desert with hot summers and mildly cool, dry winters. However, since Alkhobar is located on the Gulf's coast, the summers are exceptionally humid. During the summer Its average annual temperature is $33^{\circ} \mathrm{C}$ during the day and $22^{\circ} \mathrm{C}$ at night, with a population of 941,358 . There are only few reports of surveys conducted on the prevalence of skin diseases in Saudi Arabia. Our study is the first in the gulf region which can serve as a good reference for planning therapeutic and preventive healthcare measures in the region.

\section{Aim of the study}

To determine the prevalence and spectrum of different viral skin infections at the dermatology outpatient clinic of King Fahd Hospital of the University in Alkhobar, Kingdom of Saudi Arabia during a five-year period starting from January 2010 and ending in December 2014.

\section{MATERIALS AND METHODS}

This is a retrospective descriptive and qualitative study conducted at the dermatology outpatient clinic of King Fahd Hospital of the University in Alkhobar, Kingdom of Saudi Arabia for a five-year period extending from January 2010 until December 2014. After acquiring the Institutional Review Board approval (IRB 2015-01-186) the research was initiated. Clinical charts for all new dermatology patients who presented during that period were reviewed. Information regarding age, sex, final diagnosis was documented in a data sheet. Data was entered in the computer and analyzed through SPSS V.16 software.

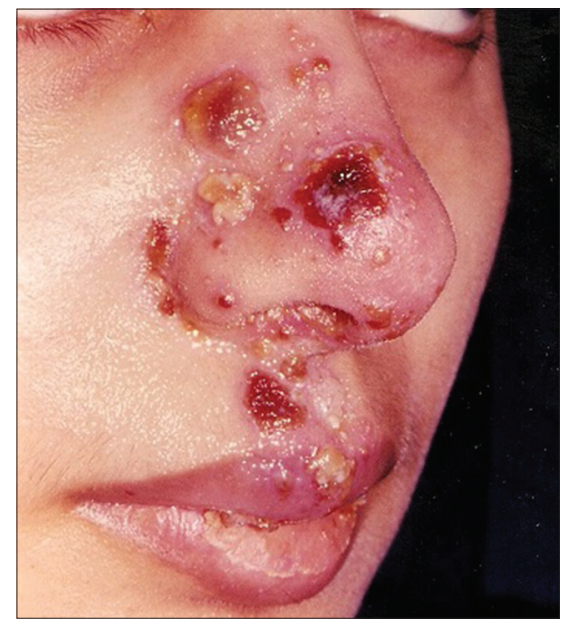

Figure 1: Herpes Zoster affecting the right maxillary branch of the trigeminal nerve in a young female patient seen at the dermatology clinics of King Fahd Hospital of the University.

\section{RESULTS}

During the study period of five years (January 2010-December 2014) different skin diseases were seen at the dermatology clinic of King Fahd Hospital of the University in Alkhobar, kingdom of Saudi Arabia. The total number of patients was 4798 seen during that period, $25.8 \%$ were in the age group of $21-30$ years, $19.5 \%$ were in the age group of $11-20$ years, and $14.1 \%$ were in the age group of 2-10 years. Female patients outnumbered male patients $(60.3 \%$ females and $39.6 \%$ males) with female to male ratio of (1.5:1). The most common encountered skin diseases were acne (16.5\%) followed by eczema (15\%), pigmentary disorders $(9.6 \%)$ and viral infections $(7.9 \%)$. A variety of viral skin infections were diagnosed including warts, herpes labialis, herpes genitalis, chicken pox and molluscum contagiosum. Among these viral skin infections warts comprised $72.5 \%$ of the total reported viral infections and majority were in the age group of 20-30 years. The second most common viral infection was molluscum contagiosum comprising $11.4 \%$ falling mainly in the age group of 1-10 years. Herpes zoster comprised $7.7 \%$ with no specific age distribution (Figs. 1 and 2). Herpes labialis was the fourth most common viral infection in

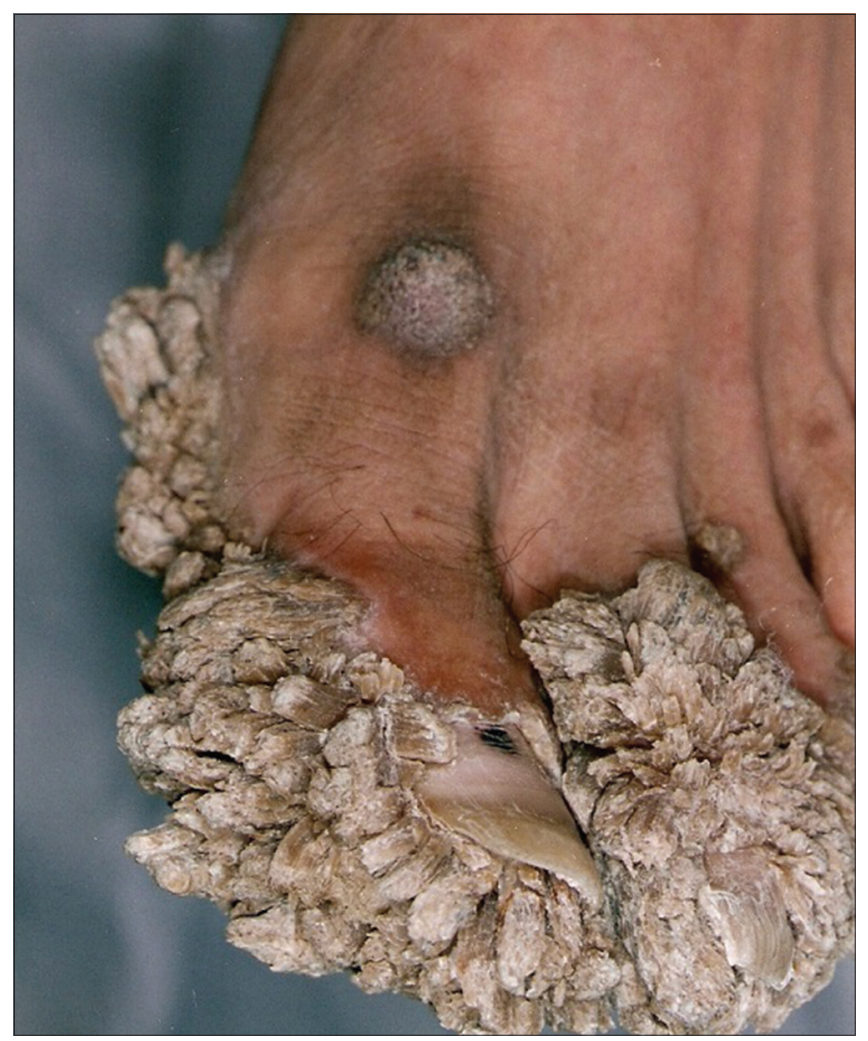

Figure 2: Giant Verruca vulgaris in an immunosuppressed kidney transplant male patient seen at the dermatology clinics at King Fahd Hospital of the University. 
Table 1: Viral infections reported at the dermatology clinics of King Fahd Hospital of the University during the study period with their percentages

\begin{tabular}{lcc}
\hline Viral infection & Number & Percentage \\
\hline Warts & 274 & 72.5 \\
Molluscum contagiosum & 43 & 11.4 \\
Herpes zoster & 29 & 7.7 \\
Herpes simplex & 18 & 4.8 \\
Chicken pox & 10 & 2.6 \\
Herpes genitalia & 3 & 0.8 \\
Measles & 1 & 0.3 \\
Total & 378 & 100 \\
\hline
\end{tabular}

our patients comprising $4.8 \%$ and occurring mainly in the age group of 20-40 years. Chicken pox and herpes genitalis were the least common reported diseases (Table 1). Warts, molluscum contagiosum, herpes zoster and chicken pox were reported more in male patients whereas herpes genitalis and measles were reported more in female patients. Herpes labialis had equal incidence in both males and females.

\section{DISCUSSION}

KFHU, being the only governmental secondary care hospital in Alkhobar, is generally exposed to a diverse inflow of patients. However, seeing as how most appointments require referral from primary health care centers, the diseases seen in the secondary hospital may not be an accurate representation of the percentages of skin disease in the population. There are undoubtedly numerous cases seen and managed in the primary health centers that did not qualify for referral to a secondary hospital. Nonetheless, KFHU receives a diverse portion of the population that paves the way for a retrospective study to be conducted.

The most commonly encountered disease group among the 4798 patients seen at KFHU during the five year period was acne $(16.5 \%)$ of which acne vulgaris was the commonest and seen mainly in the adolescent age group. Compared to the study conducted by Alsaeed et al, [2] a higher percentage of acne (22\%) was reported in their study most probably because the population consisted of school children, who are going through the changes of puberty. Most of these cases clear up on their own, hence, a portion of these children do not seek medical attention. The second most commonly encountered disease group was dermatitis and eczema (15\%) of which atopic dermatitis was the commonest. Compared to the study conducted by Alsaeed et al. [2], the decline in the prevalence of dermatitis could be attributed to the fact that most children with atopic dermatitis outgrow their conditions by the time they reach adulthood. Pigmentary disorders came in third place (9.6\%), and viral infections followed in fourth place $(7.9 \%)$. In a previous report studying pattern of skin diseases in eastern province of Saudi Arabia done by Dr. AlAkloby O, conducted at KFHU in 2002-2003, viral illnesses constituted $13.5 \%$ of all skin diseases [3]. While in 2006, Alsaeed et al. reported a prevalence rate of skin infections of $8.9 \%$ with viral infections being the second highest (27\%)[2]. Other studies done in different parts of Saudi Arabia reported viral infections to be $12.8 \%$ in Hail region and $9.1 \%$ in Najran region while in Turkey it was reported to be $7.1 \%[1,4,5]$. The difference in these percentages could be attributed to the fact that primary health centers became more involved in managing simple, uncomplicated viral diseases. For instance, Alakloby reported that warts comprised $88 \%$ of all viral illnesses seen in KFHU. That number has since been reduced to $72 \%$. This is probably attributed to the fact that primary health centers are nowadays well equipped and able to perform simple procedures to treat viral warts. Molluscum contagiosum followed as the second most common viral disease $(11.4 \%)$, and was seen predominantly in children. However, Alakloby reported that it was only seen as $2.6 \%$ of all viral infections. These varying percentages could be attributed to the increased health awareness of patients that would urge them to seek medical attention. Herpes zoster came in third (7.7\%). Alakloby reported that Herpes Zoster comprised a percentage of $6.2 \%$. The increase in percentage could be attributed to increased incidence of diabetes mellitus, advancement and increased use of immunosuppressive drugs and increased lifespan of patients who are more prone to herpes zoster. The least commonly encountered viral diseases were chicken pox and herpes genitalis. Alakloby reported similar results with $1.3 \%$ and $1.8 \%$, respectively. This could, perhaps, be due to the patients' persistent reluctance to seek medical advice with these particular diseases. With chicken pox, most patients would either choose to be treated in a primary health center or with home remedies. However, as Alakloby reported, patients with sexually transmitted diseases would choose to be treated in private institutions rather than make their records known to a governmental hospital [3].

\section{CONCLUSION}

Viral skin infections are one of the major health problems in the dermatology specialty. Further 
population based studies and public health awareness programs should be implemented to prevent and control these conditions.

\section{REFERENCES}

1. Bilgili M, Yildiz H, Sarici G. Prevalence of skin diseases in a dermatology outpatient clinic in turkey. A cross-sectional, retrospective study. J Dermatol Case Rep. 2013;4:108-12.

2. Al-Saeed W, Al-Dawood K, Bukhari I, Bahnassy A. Prevalence and pattern of skin disorders among female school children in Eastern Saudi Arabia. Saudi Med J. 2006;27:227-34.
3. Al Akloby O. Pattern of skin diseases in Eastern Saudi Arabia. Saudi Med J. 2005;26:1607-10.

4. Parthasaradhi A, Al Gufai A. The pattern of skin diseases in Hail region, Saudi Arabia. Ann Saudi Med. 1998;18:558-61.

5. Shelleh H, MD, Al-Hatiti H. Pattern of skin diseases in a hospital in southwestern Saudi Arabia. Saudi Med J. 2004;25:507-10.

Copyright by Al Anood A. Al Thukair, et al. This is an open-access article distributed under the terms of the Creative Commons Attribution License, which permits unrestricted use, distribution, and reproduction in any medium, provided the original author and source are credited.

Source of Support: Nil, Conflict of Interest: None declared. 